\title{
Chapter 126 \\ Report on Global Environment \\ Competitiveness of Sri Lanka
}

Sri Lanka is a country off the southern coast of the Indian subcontinent in South Asia. The northern part of Sri Lanka is a tropical grassland climate and the southern tropical rainforest climate. It covers 62.7 thousand of square kilometers and has a population of 20.87 million. Its GDP reaches $\$ 59.17$ billion in 2011 . Through the index system and

Score: 52.58

Rank: 39 evaluation model, the environment competitiveness index of Sri Lanka ranks at 39 in 133 countries.

Fig. 126.1 Contribution

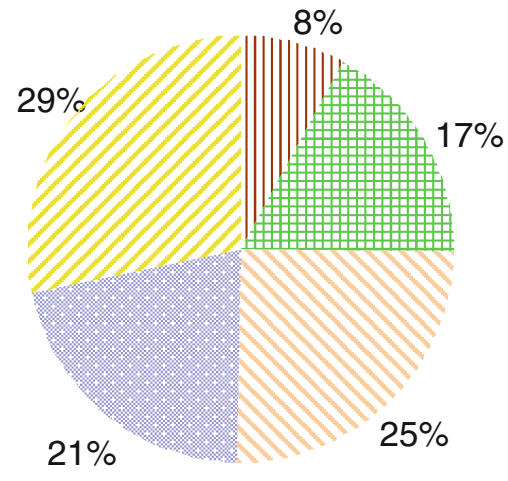

III Resource Environment Competitiveness

\# Ecological Environment Competitiveness Environment Carrying Competitiveness

Environment Management Competitiveness

Environment Harmony Competitiveness 


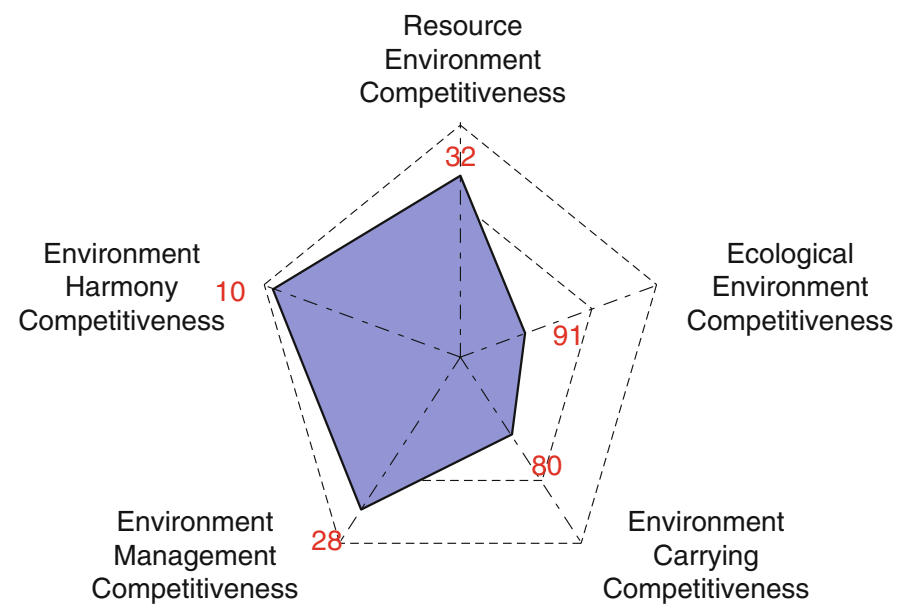

Fig. 126.2 Rank of sub-index of GEC

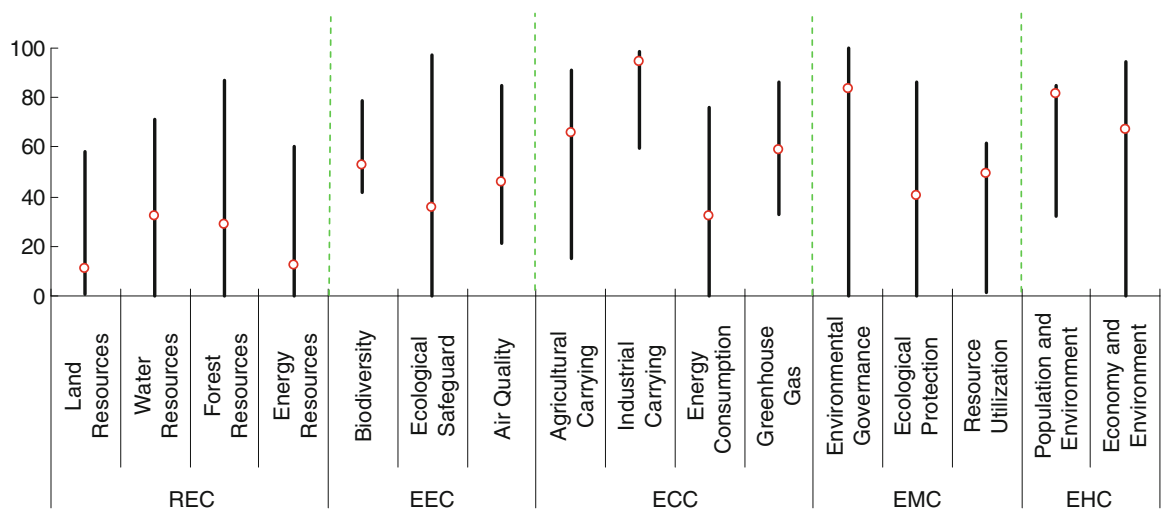

Fig. 126.3 Score and rank of the pillars of GEC

Table 126.1 Score and rank of all indicators of GEC

\begin{tabular}{|c|c|c|c|c|c|}
\hline Indicators & Score & Rank & Indicators & Score & Rank \\
\hline 1 Resource Environment & 21.33 & 32 & Groundwater & 17.88 & 44 \\
\hline Competitiveness & & & \multirow{2}{*}{$\begin{array}{l}\text { Total internal renewable } \\
\text { water resources }\end{array}$} & \multirow{2}{*}{42.46} & \multirow{2}{*}{27} \\
\hline 1.1 Land Resources & 10.72 & 78 & & & \\
\hline Land area per capita & 0.52 & 122 & 1.3 Forest Resources & 29.01 & 69 \\
\hline $\begin{array}{l}\text { Percentage of arable land } \\
\text { to total land area }\end{array}$ & 32.37 & 42 & $\begin{array}{l}\text { Growing stock in forest and } \\
\text { other wooded land }\end{array}$ & 50.12 & 100 \\
\hline Arable land per capita & 2.69 & 115 & \multirow{2}{*}{$\begin{array}{l}\text { Proportion of land area } \\
\text { covered by forest }\end{array}$} & \multirow[t]{2}{*}{34.47} & \multirow[t]{2}{*}{62} \\
\hline 1.2 Water Resources & 32.50 & 31 & & & \\
\hline Surface water & 8.95 & 39 & Forest area per capita & 0.62 & 99 \\
\hline Annual precipitation & 60.72 & 20 & 1.4 Energy Resources & 12.12 & 37 \\
\hline
\end{tabular}


Table 126.1 (continued)

\begin{tabular}{|c|c|c|c|c|c|}
\hline Indicators & Score & Rank & Indicators & Score & Rank \\
\hline Fossil energy & 0.00 & 64 & \multirow{2}{*}{$\begin{array}{l}\text { Energy consumption per } \\
\text { unit of land area }\end{array}$} & \multirow[t]{2}{*}{99.67} & \multirow[t]{2}{*}{85} \\
\hline Energy production & 0.27 & 106 & & & \\
\hline \multirow{2}{*}{$\begin{array}{l}\text { Proportion of combustible } \\
\text { renewable and waste to } \\
\text { total energy consumption }\end{array}$} & 54.85 & 23 & $\begin{array}{l}\text { Ratio of clean energy } \\
\text { consumption }\end{array}$ & 6.20 & 66 \\
\hline & \multirow[b]{2}{*}{7.05} & \multirow[b]{2}{*}{80} & Elasticity of energy & 13.57 & 91 \\
\hline $\begin{array}{l}\text { Net energy imports of the } \\
\text { energy consumption }\end{array}$ & & & \multirow{2}{*}{$\begin{array}{l}\text { consumption } \\
\text { Elasticity of electric power } \\
\text { consumption }\end{array}$} & 9.91 & \multirow[t]{2}{*}{97} \\
\hline $\begin{array}{l}2 \text { Ecological Environment } \\
\text { Competitiveness }\end{array}$ & 44.85 & 91 & & 58.88 & \\
\hline 2.1 Biodiversity & 52.57 & 121 & \multirow{2}{*}{$\begin{array}{l}\text { Growth rate of } \mathrm{CO}_{2} \\
\text { emissions }\end{array}$} & \multirow[t]{2}{*}{35.67} & \multirow[t]{2}{*}{116} \\
\hline Threatened fish species & 79.25 & 97 & & & \\
\hline Threatened mammal species & 84.24 & 108 & \multirow{2}{*}{$\begin{array}{l}\text { Growth rate of Methane } \\
\text { emissions }\end{array}$} & \multirow[t]{2}{*}{57.66} & \multirow[t]{2}{*}{73} \\
\hline Threatened plant species & 83.55 & 125 & & & \\
\hline $\begin{array}{l}\text { GEF benefits index for } \\
\text { biodiversity }\end{array}$ & 7.90 & 36 & $\begin{array}{l}\mathrm{CO}_{2} \text { emissions per unit } \\
\text { of land area }\end{array}$ & 99.76 & 72 \\
\hline 2.2 Ecological Safeguard & 35.42 & 31 & \multirow{2}{*}{$\begin{array}{l}\mathrm{CO}_{2} \text { emissions per unit } \\
\text { of energy consumption }\end{array}$} & \multirow[t]{2}{*}{65.62} & \multirow[t]{2}{*}{27} \\
\hline Terrestrial protected areas & 58.15 & 23 & & & \\
\hline Marine protected areas & 1.33 & 72 & \multirow{2}{*}{$\begin{array}{l}4 \text { Environment Management } \\
\text { Competitiveness }\end{array}$} & \multirow[t]{2}{*}{56.14} & \multirow[t]{2}{*}{28} \\
\hline 2.3 Air Quality & 46.13 & 109 & & & \\
\hline Inhalable particles (PM10) & 52.55 & 116 & \multirow{2}{*}{$\begin{array}{l}\text { 4.1 Environmental } \\
\text { Governance }\end{array}$} & 83.37 & 81 \\
\hline Particulate matter (PM2.5) & 90.91 & 30 & & \multirow{2}{*}{66.67} & \multirow{2}{*}{65} \\
\hline Index of indoor air pollution & 3.60 & 111 & \multirow{2}{*}{$\begin{array}{l}\text { Agricultural chemicals } \\
\text { regulation }\end{array}$} & & \\
\hline Nitrogen oxides emission & 68.57 & 36 & & 90.00 & 60 \\
\hline Sulfur dioxide emission & 40.48 & 76 & \multirow{2}{*}{$\begin{array}{l}\text { Percentage of the rural } \\
\text { population with access } \\
\text { to an improved water } \\
\text { source }\end{array}$} & 90.00 & 60 \\
\hline $\begin{array}{l}3 \text { Environment Carrying } \\
\text { Competitiveness }\end{array}$ & 66.31 & 80 & & & \\
\hline 3.1 Agricultural Carrying & 65.69 & 74 & Percentage of the urban & 99.00 & 51 \\
\hline $\begin{array}{l}\text { Cereal yield per unit of } \\
\text { arable land }\end{array}$ & 40.66 & 41 & $\begin{array}{l}\text { population with access } \\
\text { to an improved water }\end{array}$ & & \\
\hline Fertilizer consumption per & 79.14 & 114 & source & & \\
\hline unit of arable land & & & 4.2 Ecological Protection & 40.66 & 42 \\
\hline $\begin{array}{l}\text { Annual freshwater } \\
\text { withdrawals for }\end{array}$ & 85.60 & 116 & $\begin{array}{l}\text { Area of plantation and } \\
\text { afforestation }\end{array}$ & 0.24 & 69 \\
\hline agriculture per unit & & & Biome protect & 85.20 & 35 \\
\hline of arable land & & & Overfishing of fishing & 50.00 & 55 \\
\hline 3.2 Industrial Carrying & 94.52 & 24 & resources & & \\
\hline Net exports of goods as a & 89.33 & 25 & 4.3 Resource Utilization & 49.56 & 14 \\
\hline $\begin{array}{l}\text { percentage of GDP } \\
\text { Electric power consumption }\end{array}$ & 95.28 & 32 & $\begin{array}{l}\text { Utilization rate of water } \\
\text { resources }\end{array}$ & 0.99 & 36 \\
\hline $\begin{array}{l}\text { per unit of value added } \\
\text { of industry }\end{array}$ & & & $\begin{array}{l}\text { Percentage of total internal } \\
\text { renewable water }\end{array}$ & 88.29 & 18 \\
\hline $\begin{array}{l}\mathrm{SO}_{2} \text { emissions per unit of } \\
\quad \text { value added of industry }\end{array}$ & 99.64 & 86 & $\begin{array}{l}\text { resources to total water } \\
\text { resources }\end{array}$ & & \\
\hline $\begin{array}{l}\text { Annual freshwater } \\
\text { withdrawals for industry }\end{array}$ & 93.83 & 97 & $\begin{array}{l}\text { Percentage of agricultural } \\
\text { land to total land area }\end{array}$ & 49.20 & 71 \\
\hline $\begin{array}{l}\text { per value added } \\
\text { of industry }\end{array}$ & & & $\begin{array}{l}\text { Percentage of fossil fuel } \\
\text { energy consumption to }\end{array}$ & 59.75 & 25 \\
\hline 3.3 Energy Consumption & 32.34 & 74 & total energy consumption & & \\
\hline
\end{tabular}


Table 126.1 (continued)

\begin{tabular}{lcclcc}
\hline Indicators & Score & Rank & Indicators & Score & Rank \\
\hline $\begin{array}{l}5 \text { Environment Harmony } \\
\text { Competitiveness }\end{array}$ & 74.28 & 10 & $\begin{array}{l}\mathrm{CO}_{2} \text { emissions per capita } \\
\text { Energy consumption per } \\
\text { capita }\end{array}$ & 98.38 & 27.34 \\
$\begin{array}{l}5.1 \text { Population and } \\
\quad \text { Environment }\end{array}$ & 81.37 & 10 & $\begin{array}{l}\text { 5.2 Economy and } \\
\text { Environment }\end{array}$ & 67.18 & 51 \\
$\begin{array}{c}\text { Percentage of population } \\
\text { with access to Improved } \\
\text { sanitation facilities }\end{array}$ & 91.00 & 58 & $\begin{array}{c}\text { Land resource utilization } \\
\text { efficiency }\end{array}$ & 0.27 & 38 \\
$\begin{array}{c}\text { Motor vehicles per 1,000 } \\
\text { people }\end{array}$ & 94.44 & 38 & $\begin{array}{c}\text { Sulfur dioxide emissions per } \\
\text { unit of GDP }\end{array}$ & 88.80 & 93 \\
$\begin{array}{c}\text { Renewable internal } \\
\text { freshwater resources } \\
\text { per capita }\end{array}$ & 3.06 & 71 & $\begin{array}{c}\text { Carbon dioxide emissions } \\
\text { per unit of GDP }\end{array}$ & 91.84 & 42 \\
$\mathrm{SO}_{2}$ emissions per capita & 96.46 & 61 & $\begin{array}{c}\text { Energy consumption per } \\
\text { unit of GDP }\end{array}$ & 87.82 & 51 \\
\hline
\end{tabular}

Table 126.2 Rank distribution of the individual indicators of GEC

\begin{tabular}{lllllll}
\hline & $\begin{array}{l}\text { Number } \\
\text { of the } \\
\text { individual } \\
\text { indicators }\end{array}$ & $\begin{array}{l}\text { Rank } \\
1-10\end{array}$ & $\begin{array}{l}\text { Rank } \\
11-30\end{array}$ & $\begin{array}{l}\text { Rank } \\
31-60\end{array}$ & $\begin{array}{l}\text { Rank } \\
61-100\end{array}$ & $\begin{array}{l}\text { Rank } \\
101-133\end{array}$ \\
\hline Sub-index & 14 & 0 & 2 & 5 & 5 & 2 \\
\hline $\begin{array}{l}\text { Resource Environment } \\
\quad \text { Competitiveness } \\
\begin{array}{c}\text { Ecological Environment } \\
\quad \text { Competitiveness }\end{array}\end{array}$ & 11 & 0 & 2 & 2 & 1 & 6 \\
$\begin{array}{c}\text { Environment Carrying } \\
\quad \text { Competitiveness }\end{array}$ & 15 & 0 & 2 & 2 & 9 & 2 \\
$\begin{array}{c}\text { Environment Management } \\
\quad \text { Competitiveness }\end{array}$ & 10 & 0 & 2 & 4 & 4 & 0 \\
$\begin{array}{c}\text { Environment Harmony } \\
\quad \text { Competitiveness }\end{array}$ & 10 & 1 & 1 & 5 & 3 & 0 \\
Total & 60 & 1 & 9 & 18 & 22 & 10 \\
\hline
\end{tabular}

Open Access This chapter is distributed under the terms of the Creative Commons Attribution Noncommercial License, which permits any noncommercial use, distribution, and reproduction in any medium, provided the original author(s) and source are credited. 